\title{
The Application of Translation Brief in Enterprises and Institutions in China and Its Implications on Translator Education
}

\author{
Yanqun Zou \\ C \& E Signs Research Center \\ Beijing International Studies University \\ Beijing, China \\ e-mail: belindazou@126.com
}

\author{
Hefa Lv \\ C \& E Signs Research Center \\ Beijing International Studies University \\ Beijing, China
}

\begin{abstract}
The research surveys 139 enterprises and institutions, involving foreign companies, state-owned enterprises and private companies in various fields, on the application of translation brief in China. With the analysis of the data collected and the comparison of the study on translation brief in Denmark, the research reveals the achievement and defects in the application of translation brief in China; analyzes the unique value of translation brief in the age with diversified translation demands, and interprets the significance of translation brief in translation industry development and translator education.
\end{abstract}

Keywords-translation brief; current application; translation industry development; translator education

\section{INTRODUCTION}

Übersetzungsauftrag (translation commission, translation assignment, translation instruction), proposed by Vermeer in $1970 \mathrm{~s}$, is one of the greatest contributions of functionalism to translation. In 1996, Fraser translated it into translation brief, which is widely adopted by scholars, including Nord (1997). Translating, from a functionalist perspective, is regarded as an intentional, intercultural communicative interaction, involving the initiator, writer of ST, target reader, TT user, and translator, who needs to identify the communicative purpose to fulfill the translation task effectively and efficiently. Thus, translation brief, which provides the TT addressee(s), the prospective time and place of text reception, the medium over which the text will be transmitted, and the motive for the production or reception of the text, plays a significant role for translators to achieve the communicative function [1].

This article is a result of the project "The Application of Translation Brief in Enterprises and Institutions in China" (14Bb021) funded by Beijing International Studies University.

In the past four decades, functionalism has been applied in translation studies and practices widely, and translation brief is frequently mentioned in scholars' works. However, most works simply borrow the term with a few qualitative or empirical studies on translation brief. Jääskeläinen's (1989, 1990) studies show that the novices treat translation brief less systematically compared to professionals. Fraser (1993, 1997, 1999, 2000) maintains that translation brief is of considerable importance "in decision-making on style, register, how to deal with cultural concepts, amplification, and similar issues". She conducts a study involving 296 translators, however, only a few translators receive relevant information: addressee (s) (12\%), purpose of translation (21\%), status (text type and function) of translation (14\%) [2]. Schjoldager \& Zethesen (2003) claim that translators seem able to infer the skopos without an explicit brief by analyzing the source text, text type etc. Scholars seem hold different opinions on the use and necessity of translation brief. This study aims to explore the following questions: 1. Is translation brief (TB) necessary in translation practice? What is the current situation of the application of translation brief in China? What are the improvement and defects in application of translation brief? 2. What implications on translation industry development and translation education could be drawn from the analysis of the survey in the current context?

\section{THE SURVEY AND ANALYSIS ON THE APPLICATION OF TRANSLATION BRIEF IN ENTERPRISES AND INSTITUTIONS IN CHINA}

The C\&E Sign Research Center of Beijing International Studies University conducted a survey on the application of translation brief in China in 2014, concerning 139 enterprises and institutions (foreign companies, state-owned enterprises and private companies) in various fields, including marketing, advertisement, communications, tourism, transportation, finance, real estate, media, education \& culture industries and professional translation companies "Fig. 1". The survey collects 139 questionnaires, in which 125 are valid: 25 questionnaires from professional translation companies, 100 questionnaires from other enterprises and institutions. 


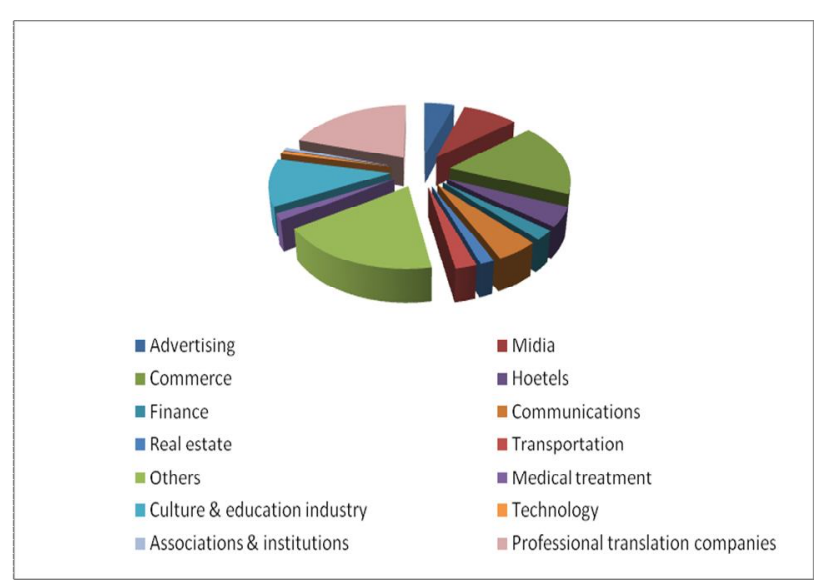

Fig. 1. Fields of Enterprises and Institutions in the Survey

A. The Awareness of the Importance of Translation Brief

With regard to the importance of TB, 86.4\% (108 respondents) consider TB very important, 12\% (15) consider TB important, and 1.6\%(2) consider TB not important, which shows that most respondents have realized the importance of TB.

\section{B. The Availability of Translation Brief}

With respect to the availability of $\mathrm{TB}, 73.6 \%$ (92) always receive $\mathrm{TB}$, and $26.4 \%$ (33) seldom receive $\mathrm{TB}$, which indicates that the offer of TB has not been institutionalized.

\section{The Access to Translation Brief}

"The person in charge of the project or department" $(42.4 \%)$ is the main access to $\mathrm{TB}, 37.6 \%$ respondents receive $\mathrm{TB}$ from the clients, $17 \%$ receive $\mathrm{TB}$ from the professional translation company, which calls for the standardization and institutionalization of the offer of TB.

\section{Translator's Initiative to Ask for Translation Brief}

Concerning "would you ask for TB if you do not receive it", $72.8 \%$ (91) say they would, while $27.2 \%$ (34) would not. The percentage of those who would ask for TB is close to the percentage of those who always receive TB $(73.6 \%)$, which demonstrates that those who always receive TB have realized the significance of TB, and would ask for it actively.

\section{E. The Choice of Translation Approach without Translation Brief}

Regarding "how to translate without TB", 62.4\% (78) respondents take ST-oriented approach, $36.8 \%$ (46) take TToriented approach, $0.8 \%$ (1) is missing.

\section{F. Implementation of Translation Brief}

As for "would you use the TB actively when you receive it", the majority of respondents $(70.4 \%, 88)$ say yes, $1(0.8 \%)$ respondent say no, and $28.8 \%$ (36) say "sometimes yes, sometimes no".

\section{G. The Identification of Text Function and Translation Purpose without Translation Brief}

Regarding "could you identify the text function without TB", $84.0 \%$ (108) say they could, while $15.2 \%$ (19) say they couldn't, 1 is missing. Concerning "could you identify the translation purpose without TB", 55.2\% (69) say they could, $44.8 \%$ (56 人) say they couldn't.

\section{H. The Awareness of the Function of Translation Brief in Translation Industry Operation}

With regard to "do you think TB is significant for the operation of translation industry", most respondents $(91.2 \%$, 114) say yes, while $8.8 \%(11)$ say no, which indicates that some respondents still hold the static view towards translation without recognizing the dynamic, contextual, and communicative features of translation, without realizing the value of $\mathrm{TB}$ for translators in choosing the translation approaches and strategies.

\section{The Constitution of Translation Brief}

Concerning the constitution of TB, the first three choices are translation criteria (73.6\%), addressee(s) (54.4\%), and translation strategies $(51.2 \%)$; while $35.2 \%$ respondents choose the motive for the production or reception of the text, $32 \%$ choose the text function, $24.8 \%$ choose the medium over which the text will be transmitted, and $13.6 \%$ choose the time and place of text reception(Figure2). Firstly, the survey indicates that the respondents pay close attention to translation criteria, addressee(s), and translation strategies; they also show concerns about the communication channels, professional standard, evaluation criterion, and call for the standardization and clarification of relevant issues. The concern for translation criterion reveals that most respondents have not developed a clear view on translation, not realizing that translation is a communication-oriented, dynamic process involving a number of factors provided by $\mathrm{TB}$, not recognizing that the translation criterion and translation strategies vary depending on the specific context and communicative purposes. While the emphasis on addressee(s) suggest some respondents realize its value in determining translation decisions. Secondly, the awareness of the importance of the time, place and medium of text reception needs to be improved. While these information may sometimes be inferred from the ST, the relevant information requires to be clarified for effective and efficient translation. Thirdly, the survey shows the prominent demand for communication between clients and translators. Fourthly, the professional requirements demand clarification. 


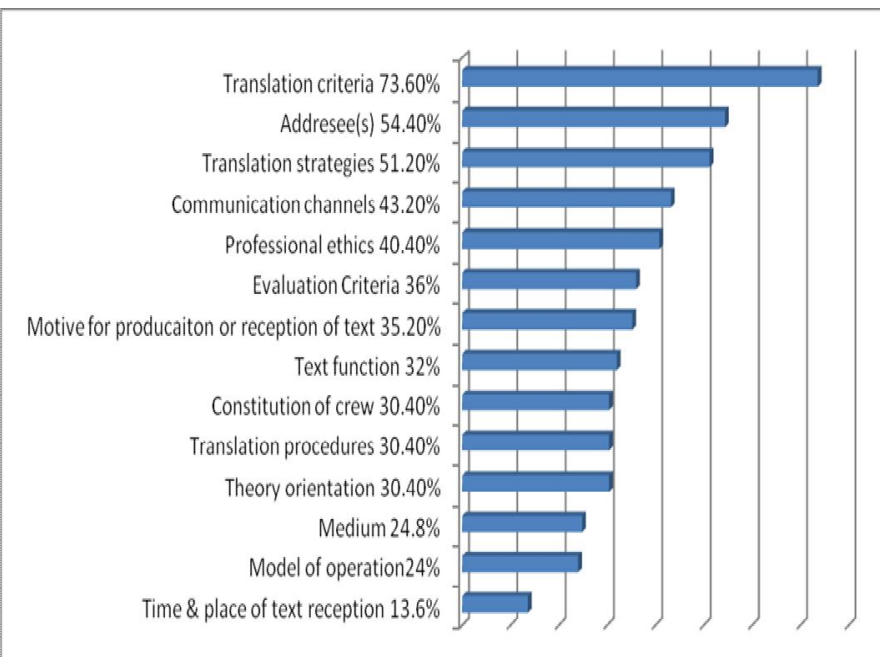

Figure 2. The factors to be included in translation brief

\section{J. Case Analysis on Practical Translation Briefs}

The majority of respondents do not provide the TB as requested, as one respondent says that $\mathrm{TB}$ in some institutions and professional companies is no more than a few words. The following two translation briefs are typical examples of TB in China.

One TB is from a company dedicated to computer games development: "The translation materials are mainly the language used in computer games, which is supposed to be local player-friendly. Foreign experts will be responsible for English and other foreign languages." Firstly, the addressee(s) are quite exclusive, and the text function, translation purpose, motive for reception of the text are lucid, and the time, place and medium of text reception could be inferred to some extent. Secondly, the TB emphasizes the target addressee(s) and target culture. Thirdly, the TB demands the target language is the translator's mother tongue. Although the translation materials are within a certain field, and some of the factors could be inferred, this TB is over-simplified, which needs to be elaborated to facilitate translators' identification of relevant issues and choice of translation approaches and strategies for the fulfillment of communicative task.

The second TB, involving preparation of translation, dos and don'ts in the process of translation, revision requirements, filing and track of customer satisfaction, is more comprehensive. What highlights the TB is: firstly, "translators should examine the ST and materials offered by client", "examine" rather than read, which suggests that translators are required to face the translation material and task critically. Secondly, this TB underlines the communication with clients, "translators are supposed to communicate with clients to solve relevant problems concerning terms and specific concepts". Thirdly, the TB stresses "the track of clients feedback", which suggests the initiative to develop the understanding about clients demands, ensure the translation quality, and make further improvement. Fourthly, the TB points out that "translators are required to keep the material confidential without revealing the information to the third party", which belongs to the professional regulations. However, the other relevant factors in TB as text function, addressee(s), time, place $\&$ medium of text acceptance, the motive for production or reception of text are overlooked, therefore, what the TB reflects is still static view towards translation without recognizing the impact of the above-mentioned factors to translation strategies.

\section{COMPARISON WITH THE RESULTS OF THE RESEARCH IN DENMARK}

Matilde Nisbeth Jensen from Aarhus School of Business completed a study entitled Professional Translators' Establishment of Skopos-A Brief Study, which aims to investigate how professional translators use TB in practice. The researcher sent out 82 questionnaires and received 47 responses, which are from professional translators with Master degree in translating and Interpreting awarded by the Danish Commerce and Companies Agency [3].

\section{A. The Awareness of the Importance of Translation Brief}

Regarding the importance of $\mathrm{TB}$, this research reveals that $85.1 \%$ respondents choose very important, $4.3 \%$ choose not very important, while $10.6 \%$ choose neutral. Compared with the survey in China, the two results are quite close, showing that most translators have realized the importance of TB.

\section{B. The Availability of Translation Brief}

The research shows that $12.8-17 \%$ respondents always receive a brief, $45 \%$ often receive a brief, $21-28 \%$ sometimes receive a brief, and none of the translators never receive a brief (The researcher asks questions about addressees and text function respectively). The survey in China reveals that $73.6 \%$ respondents in China always receive $\mathrm{TB}$, while $26.4 \%$ do not receive $\mathrm{TB}$, which suggests the percentage of $\mathrm{TB}$ availability in Denmark is higher.

\section{The Access to Translation Brief}

The research suggests that $50 \%$ respondents would ask for TB, $47.7 \%$ would not, and 1 would decline to the job. Although $50 \%$ seems a large number, there is a gap between the responses and what is done in practice-only $20 \%$ ask for $\mathrm{TB}$ in a specific task. The survey in China shows that $72.8 \%$ claim to ask for TB, which is higher than that in 2009 in Denmark, while what is done in practice needs to be investigated further.

\section{The Choice of Translation Approach without Translation Brief}

The research indicates that $67 \%$ ( 12 out of 18 ) respondents take ST-oriented approach when they do not receive $\mathrm{TB}, 22 \%$ (4/18) take TT-oriented approach, and $11 \%$

$(2 / 18)$ sometimes use one, sometimes use the other. $62 \%$ take ST-oriented approach in a practical translation task. The survey in China shows that $62.4 \%$ take ST-oriented approach, while $36.8 \%$ take TT-oriented approach, which is close to the results in 2009. ST-oriented approach is the default when translators do not receive TB, which would lead to problems in achieving the communicative tasks effectively and efficiently. 


\section{E. Implementation of Translation Brief}

Compared with the awareness of importance of TB, the research in 2009 suggests that $85.1 \%$ respondents consider TB very important, and $87 \%$ use TB actively. The survey in China reveals that $86.4 \%$ consider TB very important, while $70.4 \%$ use TB actively. The awareness of TB importance in Chinese respondents is higher, but the use of TB is less active, which leads to the following analysis: firstly, the precision and clarity of TB needs to be increased; secondly, translators' awareness of TB significance is to be improved; thirdly, translators' responsibility needs to be highlighted--they are supposed to facilitate the communication between two parties, instead of finishing the task roughly; fourthly, the communication between translators and clients demands to be enhanced thoroughly, so that TB could be used actively and communicative tasks could be fulfilled more effectively.

\section{F. The Identification of Text Function and Translation Purpose without Translation Brief}

The research in 2009, with no specific statistics on this issue, points out that many could establish the function of translation, and quite a few find it difficult sometimes, when they translate using a neutral receiver group or by addressing the TT at a broad international group. The survey in China displays that $44.8 \%$ could not establish the motive for translation production, and $15.2 \%$ could not establish the text function, which would bring serious barriers for translators to fulfill the expected communicative effect.

\section{THE ANALYSIS ON THE CURRENT APPLICATION OF TRANSLATION BRIEF IN CHINA AND ITS IMPLICATIONS}

TB was originally proposed for translator training, since the experienced translator will be able to infer the skopos even without a brief, by just taking the translational situation into account [4]. But the surveys reveal that the fact sometimes is different from what scholars expected, which highlights the value and significance of TB in translation practice, translation industry development, and translator education in the ever changing society with updating information, technologies, and various demands in translation.

\section{A. The Analysis on the Current Application of Translation Brief in China and its Unique Value in the Current Context \\ 1) The analysis on the current application of translation brief in China}

The survey on TB application in China reveals that the consciousness of the necessity and importance of TB has been greatly improved; and some enterprises and institutions apply TB to enhance the translation process and quality management effectively; furthermore, most translators use TB actively to identify text function and motive for translation production in order to optimize the resources and improve translation quality and communicative effect. The introduction of translation theories, the improvement in translation management, and progress in translator education bring the development in translation practice and industry to some extent.
The survey also exposes some problems in TB use. The operation of translation in certain enterprises and institutions is far from being standardized or institutionalized-the socalled TB is no more than a few words, or no TB offered at all, some even equal TB to translation regulations, which would lead to the uncertainty of translation quality. TB plays a significant role in boosting the standardization of translation management.

What deserves our attention is the concern about communication channels, professional ethics, evaluation principles, translation procedures, and operation models, which exhibit the complexity of translation, and the demand for a more comprehensive, elaborate TB.

The survey also compares the differences between enterprises and institutions, foreign and state-owned companies, professional translation companies and other enterprises \& institutions, renowned translation companies and common translation companies on TB use, the results do not show great significance. However, the differences between different fields on TB use are significant: the TB availability of $\mathrm{TB}$ in advertising industry is higher than that in professional translation companies; $50 \%$ respondents from advertising industry take ST-oriented approach, and 50\% take TT-oriented approach; the majority of respondents from real estate take ST-oriented approach; $57 \%$ in media industry take TT-oriented approach. The addressee(s), text function, and clients requirements are the decisive factors, which will be elaborated in forthcoming papers.

2) The unique value of translation brief in the current context

The development of a more comprehensive and elaborate $\mathrm{TB}$ is the call of the age. With the constant development of disciplines and technologies, especially information technology, translators are facing a world with constantly updating information, diversified demands from clients, specifically segmented addressee(s) and communicative purposes, various medium, different macrocontexts and micro-circumstances, and with unpredictable problems to be solved in translation tasks, which highlight the unique function of TB to clarify the relevant information to cater for the huge diversity in the current market and the constantly developing society. What translation, especial applied translation, in the current context stresses are the contextual, communicative, functional features, which demands a comprehensive TB to facilitate the achievement of the complex, non-routine tasks with varying requirements. Consequently, a comprehensive, elaborate $\mathrm{TB}$ is of considerable significance in promoting the translation quality and communicative effect in the current context.

\section{B. The Implications}

1) A comprehensive translation briefformat is supposed to be developed and institutionalized to boost the translation industry development and translator education.

Being a kind of communicative interactions, translations take place in situations that are limited in time and space [5], 
which make translation a dynamic process, a process of construction of meaning with ST in the intercultural context [6].

Vermeer stresses the great importance of the TT by proclaiming the dethronement of the ST and regarding it as a mere offer of information" and the translator's "raw material" [7]. Nord holds the same view. While the motives of the two parties in intercultural communication are dynamic, what translators are supposed to realize is not the transfer of static meaning of ST, but the communicative task and effect, while $\mathrm{TB}$ is the prerequisite and basis to achieve the expected function.

Furthermore, "translational intention may or may not be similar to the intention guiding the original sender or text producer in the production of the source text, which is an intentional communicative interaction it itself' [5]. Translators introduce the linguistic and cultural information offered by ST to the TT addressee(s) with certain communicative purpose, while the choice of the information, translation strategies and TT style depend on the client and addressees' expectation and needs [8]. The translator need as much knowledge as possible about the communicate e purposes the TT is supposed to achieve for the addresses in their communicative situation. These details are explicitly or implicitly defined in the translation brief [5]. TB is the foundation for translators to choose the appropriate translation strategies, to fulfill the communicative task, and to optimize the communicative effect.

Consequently, a comprehensive, elaborate TB format needs to be developed and institutionalized for relevant agencies and translators to follow with communication channels, translation procedures, and other necessary information included, to boost the translation industry development and translator education. As Nord says, "Translating without clear instructions is like swimming without water" [7].

2) Translation brief is supposed to be integrated in the course of translation education.

Both translators and clients or initiators are supposed to improve the consciousness on the importance of TB, which determines the translation approaches, strategies and the fulfillment of communicative task, and the emphasis on TB should be highlighted in translator education. Firstly, TB is to be integrated in the course of translation education: in the preparation of translation, in the process of translating, and revision, referring to the information offered by TB to help students realize the function and essence of translation, the role of translator in the current context with diverse translation demands. Secondly, TB is to be incorporated in translation teaching, practice and test. In translation teaching and practice, teachers are supposed to adopt the process-oriented approach, and guide students to develop a dynamic view towards translation, and analyze the differences between ST and TT in terms of factors mentioned in TB, and differences between target texts with different translation briefs, to help students realize the impacts of factors mentioned in TB to TT, and to facilitate students to cultivate the habit of referring to $\mathrm{TB}$ before, in and after translation to enhance the translation effectiveness and efficiency. In translation tests, the central information of TB--the addressee(s), the time, place \& medium of text reception, the motive for the production or reception of the text-is supposed to be provided, so that students could choose the appropriate approaches and cultivate the habit of referring to TB. The integration of TB in these activities will promote students' awareness of the significance of TB, which helps students - the prospective translators and translation industry participants--develop the initiative to ask for TB if they do not receive one, or provide TB actively, and boosts the standardization and institutionalization of TB.

3) Communication between translators and clients needs to be promoted.

TB is a bridge between clients and translators to fulfill the communicative task, however, TB sometimes could not offer sufficient information in the current context with various demands in translation, thus, communication between the two parties involved becomes a necessity. Besides the inclusion of communication channels in $\mathrm{TB}$, the two parties are encouraged to communicate thoroughly in different respects, including terminologies, specific concepts, resources and other necessary information. Besides it is the translator's responsibility to educate clients so they can learn to provide the necessary information and other available data (Nord, 2006, p.30). Furthermore, it is one of translation education's tasks to cultivate students' communicative competence and metacognitive competence to enhance communication effectiveness.

4) The role and responsibility of translators need to be elaborated.

With the information offered by $\mathrm{TB}$, translators are supposed to see not only texts but also people behind texts, and the communication between people in different cultures with a number of dynamic factors; translators are required to realize the dynamic, contextual and communicative features of translation, and improve their metacognitive competence to meet the requirements of the ever changing society with constantly updating technologies and information. What translators need to develop are not confined within linguistic and encyclopedic competences, but also instrumental, translation knowledge, and strategic competences(PACTE); what translators need to learn is not only static declarative knowledge, but also dynamic procedural and conditional knowledge to improve the competence to analyze and solve problems encountered in translation, so that they could optimize the resources at disposition, explore effective and efficient strategies, monitor the process and regulate if necessary, and achieve the communicative effect. Translators are required to reflect on what they are doing and become lifelong learners to achieve and optimize the communicative effect in the constantly developing society.

TB plays a unique role in enhancing translation effectiveness and efficiency, in boosting translation industry development, and in improving translation education in the current context. 


\section{REFERENCES}

[1] Nord, C. Text Analysis in Translation. Amsterdam: Rodopi. Transl. from the German by C. Nord and P. Sparrow. German original(11988) Textanalyse und Übersetzen. Heidelberg: Julius Groos , 1991.

[2] Fraser, J. "The broader view: How freelance translators define translation competence". In C. Schäffner \& B. Adab Developing translation competence. Shanghai: Shanghai Foreign Language Education Press, 2012(2000), pp.53-54.

[3] Jensen M. N. Professional translators' establishment of skopos- a 'brief' study. Aarhus School of Business, Institut for Sprog og Erhvervskommunikation, 2009.

[4] Vermeer, H. J. "Skopos and commission in translation action". In A. Chesterman(ed.) Readings in translation. Oy Finn Lectura Ab. 1989, pp. 182.

[5] Nord, C. "Defining translation functions. The translation brief as a guideline for the trainee translator". In W. Lorscher(ed.) Ilha do Desterro, no. 33, pp. 43-46.

[6] Peng L. Translation context. Chinese Dictionary on Translation. Shanghai: Shanghai Foreign Language and Education Press. 2011.

[7] Nord, C. Translating as a Purposeful Activity. St. Jerome Publishing, 1997, pp. 37-48.

[8] Fang M. "Applied translation in China: Theory and Teaching". China Translator Journal. 2011, vol. 3, pp. 34-36. 